\title{
PARETO OPTIMAL SOLUTION OF MULTIOBJECTIVE SYNTHESIS OF ROBUST CONTROLLERS OF MULTIMASS ELECTROMECHANICAL SYSTEMS BASED ON MULTISWARM STOCHASTIC MULTIAGENT OPTIMIZATION
}

Purpose. Developed the method for solving the problem of multiobjective synthesis of robust control by multimass electromechanical systems based on the construction of the Pareto optimal solutions using multiswarm stochastic multi-agent optimization of particles swarm, which reduces the time of determining the parameters of robust controls multimass electromechanical systems and satisfy a variety of requirements that apply to the work of such systems in different modes. Methodology. Multiobjective synthesis of robust control of multimass electromechanical systems is reduced to the solution of solving the problem of multiobjective optimization. To correct the above problem solving multiobjective optimization in addition to the vector optimization criteria and constraints must also be aware of the binary preference relations of local solutions against each other. The basis for such a formal approach is to build areas of Pareto-optimal solutions. This approach can significantly narrow down the range of possible solutions of the problem of optimal initial multiobjective optimization and, consequently, reduce the complexity of the person making the decision on the selection of a single version of the optimal solution. Results. The results of the synthesis of multi-criteria electromechanical servo system and a comparison of dynamic characteristics, and it is shown that the use of synthesized robust controllers reduced the error guidance working mechanism and reduced the system sensitivity to changes in the control parameters of the object compared to the existing system with standard controls. Originality. For the first time, based on the construction of the Pareto optimal solutions using a multiswarm stochastic multi-agent optimization particle algorithms improved method for solving formulated multiobjective multiextremal nonlinear programming problem with constraints, to which the problem of multiobjective synthesis of robust controls by multimass electromechanical systems that can significantly reduce the time to solve problems and meet a variety of requirements that apply to the multimass electromechanical systems in different modes. Practical value. Practical recommendations on reasonable selection of the target vector of robust control by multimass electromechanical systems. Results of synthesis of electromechanical servo system shown that the use of synthesized robust controllers reduced the error guidance of working mechanism and reduce the system sensitivity to changes of plant parameters compared to a system with standard controls. References 9, figures 3.

Key words: multimass electromechanical system, multiobjective synthesis, multiswarm stochastic multiagent optimization, Pareto optimal solution.

Усовериенствован метод многокритериального синтеза робастного управления многомассовыми электромеханическими системами на основе построения Парето-оптимальных решений и с учетом бинарных отношений предпочтения локальных критериев с помощью алгоритмов многороевой стохастической мультиагентной оптимизации, что позволяет существенно сократить время решения задачи и удовлетворить разнообразным требованиям, которые предвявляются к работе систем в различных режимах. Приведены результаты сравнений динамических характеристик электромеханических систем с синтезированными регуляторами. Библ. 9, рис. 3.

Ключевые слова: многомассовая электромеханическая система, многокритериальный синтез, многороевая стохастическая мультиагентная оптимизация, Парето-оптимальное решение.

Introduction. When creating new generations of technology and new technologies, automatic control systems are required that can provide high accuracy in the presence of intense driving and disturbing influences, as well as changes in the structure and parameters of systems during their operation. Such control systems usually have very diverse and often contradictory requirements for the operation of the system in various modes and under various external influences: stepwise, linearly varying, harmonic, random, etc. $[8,9]$, so that the problem of synthesizing such systems in its formulation is multicriteria.

Problem definition. Multicriterion synthesis of robust control of multimass electromechanical systems can be reduced [8] to the solution of a multicriteria nonlinear programming problem in which the vector objective function

$$
f(X)=\left[f_{1}(X), f_{2}(X) \ldots f_{k}(X)\right]^{T}
$$

and constraints on controls and variables

$$
G(X) \leq G_{\max }, H(X)=0 .
$$

Components $f_{i}(X)$ of the vector criterion (1) are local optimization criteria for a multimass electromechanical system, such as the time of the first matching, the time of regulation, overshooting, etc.

The components of the vector of the required parameters $X$ are the elements of the weight matrices, with the help of which the target vector of the robust control is formed [8].

The calculation of the vector objective function (1) and constraints (2) has an algorithmic character and is related to the solution of the problem of synthesizing robust regulators with the help of which the target vector $H_{\infty}$ is minimized, and reduces to the calculation of four algebraic Riccati equations [8] for computing the robust regulator and Robust observer within the framework of four Riccati approach to the synthesis of robust systems. The solution of the problem of the synthesis of anisotropic regulators, by means of which the average anisotropy of the system is minimized, is reduced to the calculation of four algebraic Riccati equations, the

(C) T.B. Nikitina 
Lyapunov equation, and a special expression for calculating the anisotropy level of the input signal [9].

Solving the multicriteria optimization problem by collapsing the vector criterion into a scalar one using weight factors [4, 5] actually replaces the original problem of solving multicriteria optimization for the problem of choosing weight factors. Often the problem of correctly determining the weight factors in complexity is many times greater than the original problem of solving the multicriteria optimization problem, since to determine the weight factors it is necessary to solve the problem of scalar optimization repeatedly at the routine load of the decision-maker. In addition, the scalar target function obtained as a result of this transformation has sections of the «plateau» and «ravine» type, which requires special algorithms for solving it, while the scalar components of the vector objective function of the original multicriteria optimization problem are sufficiently smooth.

To date, the development of the theory of the correct solution of the original multicriteria optimization problem is completed on the basis of constructing Pareto-optimal solutions without the procedure for minimizing local criteria. In order to find a global optimum, in addition to specifying a vector objective function and constraints, it is also necessary to specify the binary relations of preferences for local optimization criteria that are components of the initial vector optimization criterion. To solve such a problem on the basis of Pareto-optimal solutions, algorithms of multi-stochastic stochastic multiagent optimization are being used most successfully [1-3]. In this case, the problems of multicriteria optimization with the limitations $[6,7]$ are of greatest complexity. Let us consider one of the variants of constructing such an algorithm on the basis of nonlinear control laws.

The goal of the paper is improvement of the method of solving the problem of multicriteria synthesis of robust control multimass electromechanical systems by constructing a Pareto-optimal solutions, and taking into account the binary preference relations of local optimization criteria using multiswarm stochastic multiagent optimization of particle swarm, which reduces the time of determining the parameters of robust controllers multimass electromechanical systems and meet the diverse requirements that apply to the such systems operation in different modes.

Search for the Pareto set on the basis of multiswarm stochastic multiagent optimization. In order to correctly solve the multicriteria optimization problem, in addition to the vector optimization criterion (1) and constraints (2), it is also necessary to have information about the binary relations of preferences of local solutions relative to each other [5]. The basis of such a formal approach is the construction of Pareto-optimal solutions. This approach allows us to significantly narrow the range of possible optimal solutions of the original multicriteria optimization problem and, consequently, to reduce the complexity of the person making the decision to choose the only variant of the optimal solution.

The problem of finding the minimum of the local criterion $f_{i}(X)$ in the space under consideration, as a rule, is multiextremal, containing local minima and maxima, therefore, it is expedient to use the stochastic multi-agent optimization algorithms to solve it [2]. Consider the algorithm for finding the set of Pareto-optimal solutions of multicriteria non-linear programming problems on the basis of stochastic multi-agent optimization. To date, a large number of algorithms have been developed for optimizing the swarm of particles - PSO algorithms based on the idea of collective intelligence of a swarm of particles, such as gbest PSO and lbest PSO algorithms [6]. The use of stochastic multi-agent optimization methods for solving multicriteria problems today causes certain difficulties and this direction continues to develop intensively [7]. To solve the original multicriteria nonlinear programming problem (1) with constraints (2), we construct an algorithm for stochastic multi-agent optimization based on the set of particle swarms, the number of which is equal to the number of components of the vector optimization criterion. In the standard optimization algorithm for swarms of particles, the particle velocities are varied according to linear laws [6]. To increase the speed of finding a global solution, special non-linear algorithms of stochastic multi-agent optimization proposed in [1] have recently been extended in which the motion of the $i$-th particle of the $j$-th swarm is described by the following expressions

$$
\begin{gathered}
v_{i j}(t+1)=w_{j} v_{i j}(t)+c_{1 j} r_{1 j}(t) H\left(p_{1 j}-\varepsilon_{1 j}(t)\right)\left[y_{i j}(t)-x_{i j}(t)\right]+ \\
+c_{2 j} r_{2 j}(t) H\left(p_{2 j}-\varepsilon_{2 j}(t)\right)\left[y_{j}^{*}(t)-x_{i j}(t)\right], \\
x_{i j}(t+1)=x_{i j}(t)+v_{i j}(t+1),
\end{gathered}
$$

where $x_{i j}(t), v_{i j}(t)$ are the position and velocity of the $i$-th particle of the $j$-th swarm; $c_{1}$ and $c_{2}$ are the positive constants that determine the weights of the cognitive and social components of the velocity of the particle; $r_{1 j}(t)$ and $r_{2 j}(t)$ are the random numbers from the range $[0,1]$, which determine the stochastic component of the velocity component of the particle. Here $y_{i j}(t)$ and $y_{j}^{*}$ are the best local - lbest and global - gbest positions of the $i$-th particle, found respectively by only one $i$-th particle and all the particles of the $j$-th swarm. The use of the inertia coefficient $w_{j}$ makes it possible to improve the quality of the optimization process.

The Heaviside function is used as a function of switching $H$ the particle motion to the local $y_{i j}(t)$ and the global $y_{j}^{*}(t)$ optimum, respectively. The parameters for switching cognitive $p_{1 j}$ and social $p_{2 j}$ components of the particle velocity to the local and global optimum, respectively; The random numbers $\varepsilon_{1 j}(t)$ and $\varepsilon_{2 j}(t)$ determine the parameters of the particle motion switching, respectively, to the local and global optimum. If $p_{1 j}<\varepsilon_{1 j}(t)$ и $p_{2 j}<\varepsilon_{2 j}(t)$, then the velocity of the $i$-th particle of the $j$-th swarm does not change at step $t$ and the particle moves in the same direction as in the previous optimization step.

With the help of separate $j$-th swarms (3), (4), optimization problems for the scalar criteria $f_{i}(X)$ which are components of the vector optimization criterion (1), are solved. To find a global solution of the original multicriteria problem in the course of searching for optimal solutions of local criteria, individual swarms exchange information among themselves. In this case, to calculate the velocity of the particles of one swarm, 
information is used about the global optimum found by the particles of another swarm, which makes it possible to isolate all potential Pareto-optimal solutions. For this purpose, at each step $t$ of the motion of the $i$-th particle of the $j$-th swarm, the preferences functions of the local solutions obtained by all swarms are used. The solution $X_{j}^{*}(t)$ obtained by optimizing the objective function $f_{i}(X)$ with the $j$-th swarm is preferable to the solution $X_{k}^{*}(t)$ obtained by optimizing the objective function $f_{j}(X)$ with the help of the $k$-th swarm, i.e. $X_{j}^{*}(t) \succ X_{k}^{*}(t)$, if the binary preference condition is met. Moreover, as the global optimal solution $X_{k}^{*}(t)$ of the $k$-th swarm, a global solution $X_{j}^{*}(t)$ obtained by the $j$-th swarm is used that is preferable to the global solution $X_{k}^{*}(t)$ of the $k$-th swarm based on the preference relation.

In fact, with this approach, the basic idea of the method of successive narrowing of the field of compromises is realized: from the initial set of possible solutions, based on information about the relative importance of local solutions, all Pareto-optimal solutions that can not be selected according to the available information on binary preferences of local criteria are deleted successively. Removal is carried out until a globally optimal solution is obtained. As a result of applying this approach, no potentially optimal solution will be removed at each step of the contraction.

Usually, the initial position of the swarm agents is randomly assigned, and then the swarm moves to the global optimum starting from this position, which characterizes the stochastic properties of the optimization algorithm. The number of agents in the swarm can remain constant, or they will change as the swarm moves. With a constant number of swarm agents, the swarm topologies of the «ring», «square», «star» and «pyramid» types are most often used. In particular, the gbest PSO and lbest PSO algorithms use the swarm topologies of the «star» and «ring» type. If you change the number of agents in a swarm, the initial number of swarm agents is randomly assigned, and then from this number of agents, a random formation of a new number of agents and a new swarm topology begins.

When moving, the swarm particles try to improve the solution they found earlier and exchange information with their neighbors, thereby finding a global optimum for the fewer number of iterations. The advantage of these methods over the classical gradient optimization methods is that they do not require the calculation of the derivatives of the objective function, they are practically insensitive to the proximity of the initial position to the desired optimal solution, and it makes it easier to take into account various constraints in finding the global optimum.

Results of computer modeling. As an example, we consider the transient processes in the electromechanical servo system [9] with synthesized robust regulators. One of the characteristic modes of operation of the electromechanical servo system under consideration is the development of a given linearly varying angle of rotation of the working mechanism. To ensure a zero steady-state error of the system in this mode, a second order of the system's astaticism is required for the determining influence. In the existing system, PD controllers are used, since the introduction of the integral component leads to the emergence of undamped oscillations in the mode of working out the given angles of the position of the working member due to the presence of dry friction on the shafts of the drive motor and the working member. With the help of robust regulators it was possible to ensure a stable operation of the system taking into account all the essential nonlinearities inherent in the elements of this system when two integrating links are introduced into the control loop.

We perform a study of the sensitivity of such a robust system with second-order astatism, taking into account all the nonlinearities for three different values of the moment of inertia of the working member, which varies during the operation of the system.

Fig. 1 shows the transient processes: a) the rotation angle $\varphi(t)$ and b) the rotation speed $\omega_{m}(t)$ of the working mechanism when hovering at transfer speeds (35 deg/s).
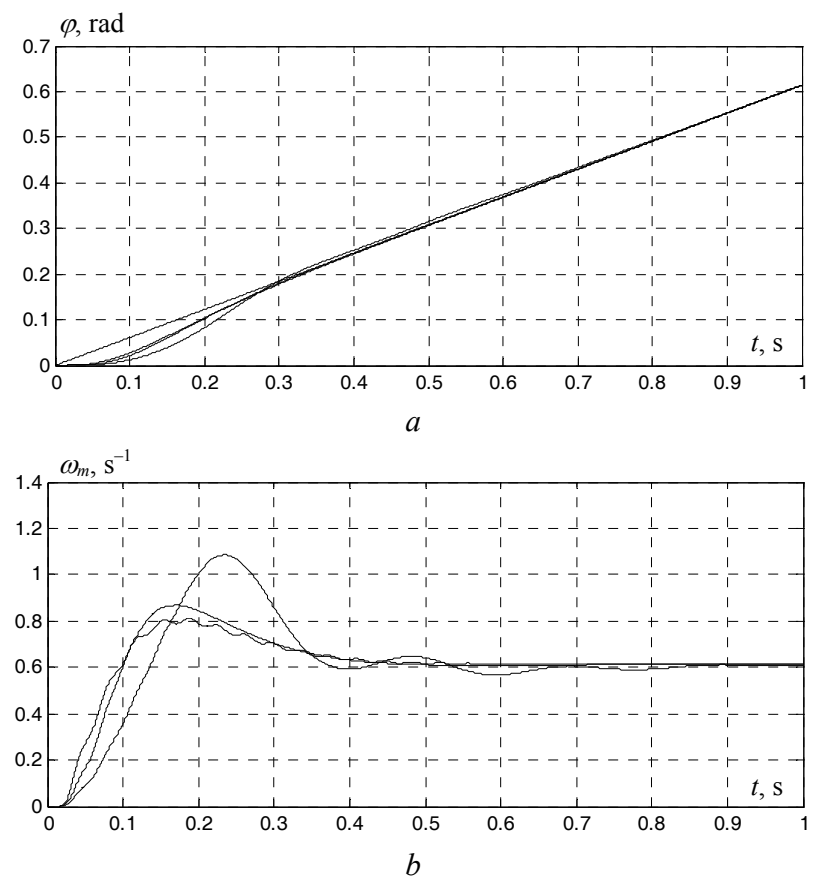

Fig. 1. Transient processes of:

a) rotation angle $\varphi(t) ; b)$ rotation speed $\omega_{m}(t)$ of the working mechanism when hovering at transfer speeds $(35 \mathrm{deg} / \mathrm{s})$

The steady error in working out the given linearly varying rotation angle of the working mechanism is zero and, consequently, the synthesized system has a secondorder astatism. Note that during the first $0.02 \mathrm{~s}$. The working member remains stationary, since during this time the drive motor is stuck and then the shaft connecting the drive motor and the operating member is angled such that the elastic moment becomes greater than the dry friction moment of the working member. When the moment of inertia of the working mechanism changes, the transient processes change insignificantly, which confirms the weak sensitivity of the system being synthesized, and the steady-state velocities of the working mechanism coincide. 
Fig. 2 shows the transient processes of the same state variables when the working mechanism is guided at low velocities $(0.5 \mathrm{deg} / \mathrm{s})$. At the same time, the working mechanism moves with jerks and with stops, but the steady error of the system is practically zero. It should be noted that in this mode the working element remains stationary during the first $0.08 \mathrm{~s}$, which is four times longer than when the working member moves with a transfer speed, as shown in Fig. 1.
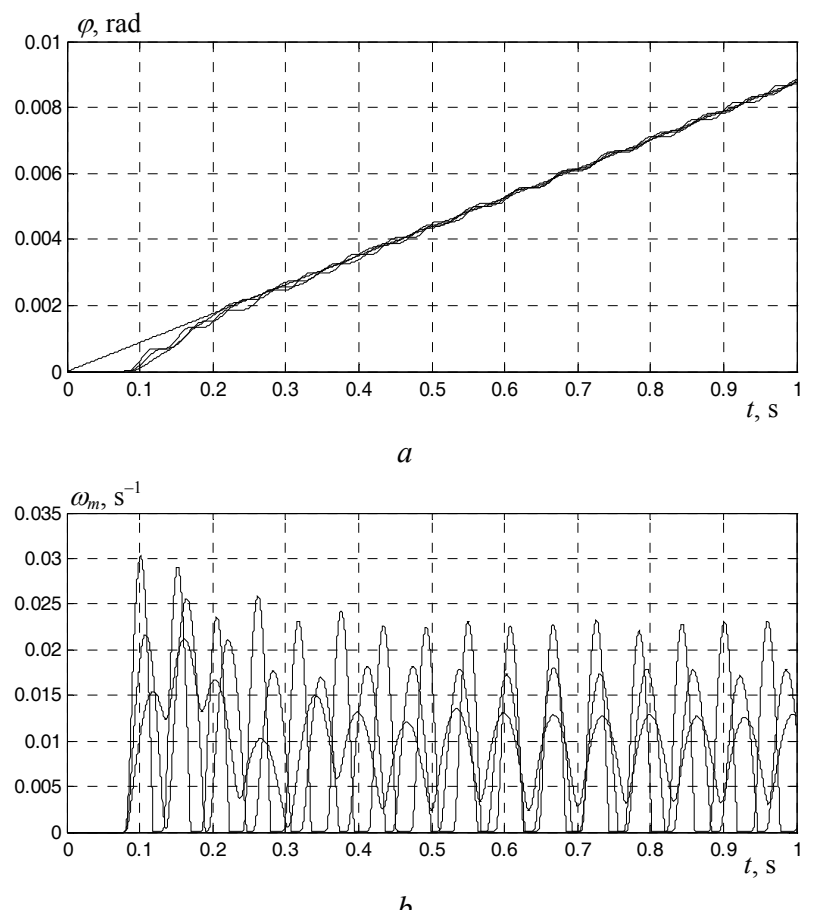

Fig. 2. Transient processes of:

a) rotation angle $\varphi(t) ; b)$ rotation speed $\omega_{m}(t)$ of the working mechanism when hovering at low speeds $(0.5 \mathrm{deg} / \mathrm{s})$

Fig. 3 shows the transient processes of the same state variables when the working mechanism is guided at super small - creeping speeds $(0.02 \mathrm{deg} / \mathrm{s})$. We note that this mode of operation determines the potential accuracy of the electromechanical servo system under consideration and, in general, characterizes its tactical and technical characteristics [9]. In this mode, the working element remains stationary for the first $0.3 \mathrm{~s}$, which is 15 times longer than when the working member moves at transfer speed. Such a long delay in the beginning of the movement of the working element is caused by the required time required for the appearance of an error in working out a given angle of the position of the working member and the generation of the corresponding motor moments and the moment of elasticity of the shaft connecting the drive motor with the working member necessary for friction, first the drive motor, and then Working member of the electromechanical servo system. At the same time, the working mechanism moves in jerks and contains sections like stopping, moving forward, stopping, moving backwards, and the system error in the steady state oscillates with respect to the zero value with an amplitude of $5 \cdot 10^{-4} \mathrm{rad}$.

The results of comparisons of the dynamic characteristics of the servo electromechanical system have shown that the use of synthesized robust controllers has made it possible to reduce the error in setting the working mechanism and to reduce the sensitivity of the system to changes in the parameters of the control object in comparison with the existing system with typical regulators.
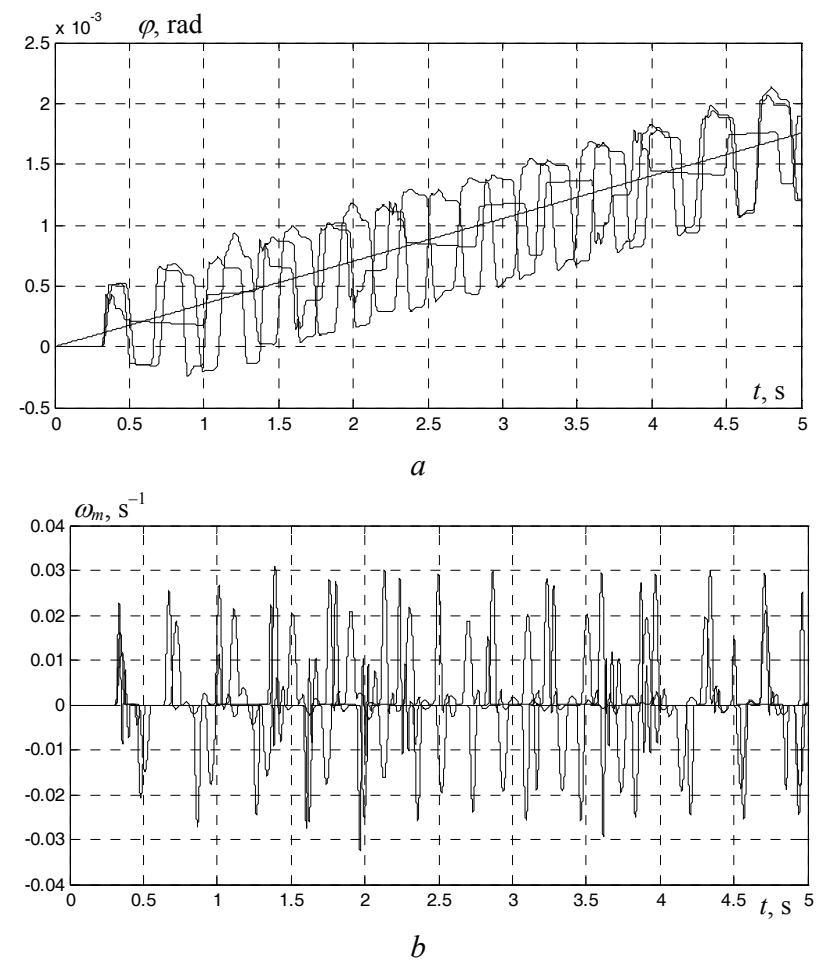

Fig. 3. Transient processes of:

a) rotation angle $\varphi(t) ; b)$ rotation speed $\omega_{m}(t)$ of the working mechanism when hovering at super small - creeping speeds $(0.02 \mathrm{deg} / \mathrm{s})$

\section{Conclusions.}

On the basis of the construction of the Paretooptimal solutions, and taking into account the binary preference relations of local criteria using stochastic multi-agent optimization particle by multiswarm algorithms improved method for solving formulated multiobjective multiextremal nonlinear programming problem with constraints, to which the problem of multicriteria synthesis of robust controllers multimass electromechanical systems that can significantly reduce the time for solving the problem and satisfy different requirements that apply to the work multimass electromechanical systems in various modes. It is shown that the use of synthesized robust controllers possible to reduce the pointing error of the working mechanism and reduce the sensitivity of the system to change control parameters of the object compared to a typical system regulators.

\section{REFERENCES}

1. Abido M.A. Two-level of nondominated solutions approach to multiobjective particle swarm optimization. Proceedings of the 9th annual conference on Genetic and evolutionary computation - GECCO'07. 2007, pp. 726-733. doi: 10.1145/1276958.1277109.

2. Clerc M. Particle Swarm Optimization. London, ISTE Ltd., 2006. 244 p. doi: 10.1002/9780470612163. 
3. Fieldsend Jonathan E., Singh Sameer. A multi-objective algorithm based upon particle swarm optimization, an efficient data structure and turbulence. Proceedings of the 2002 U.K. Workshop on Computational Intelligence, 2002, pp. 37-44.

4. Gazi V., Passino K.M. Swarm Stability and Optimization. Springer, 2011. 318 p. doi: 10.1007/978-3-642-18041-5.

5. Hu Xiaohui, Eberhart R. Multiobjective optimization using dynamic neighborhood particle swarm optimization. Proceedings of the 2002 Congress on Evolutionary Computation. CEC'02 (Cat. No.02TH8600). doi: 10.1109/cec.2002.1004494.

6. Nor Azlina Ab. Aziz, Mohamad Yusoff Alias, Ammar W. Mohemmed, Kamarulzaman Ab. Aziz. Particle Swarm Optimization for constrained and multiobjective problems: a brief review. International Conference on Management and Artificial Intelligence IPEDR. Bali, Indonesia, no.6, pp. 146-150.

7. Zizler Eckart. Evolutionary algorithms for multiobjctive optimizations: methods and applications. Ph.D. Thesis Swiss Federal Institute of Technology, Zurich, 1999. 122 p.
8. Nikitina T.B. Mnogokriterial'nyj sintez robastnogo upravlenija mnogomassovymi sistemami [Multicriterion synthesis of robust control by multimass systems]. Kharkiv, Kharkiv National Automobile and Highway University Publ., 2013. 432 p. (Rus).

9. Nikitina T.B. Multiobjective synthesis of robust control by multimass electromechanical systems based on Pareto-optimal solution. Electrical engineering \& electromechanics, 2015, no.1, pp. 29-35. (Rus). doi: 10.20998/2074-272X.2015.1.06.

Received 20.11.2016

T.B. Nikitina, Doctor of Technical Science, Professor, Kharkov National Automobile and Highway University, 25, Petrovskogo Str., Kharkov, 61002, Ukraine,

e-mail: tatjana55555@gmail.com

How to cite this article:

Nikitina T.B. Pareto optimal solution of multiobjective synthesis of robust controllers of multimass electromechanical systems based on multiswarm stochastic multiagent optimization. Electrical engineering \& electromechanics, 2017 , no.2, pp. 34-38. doi: 10.20998/2074-272X.2017.2.05. 